\title{
Identification of Enterovirus C105 for the first time in New Zealand
}

\author{
Angela Todd, a Susan Taylor ${ }^{b}$ and Q Sue Huang ${ }^{a}$ \\ Correspondence to Angela Todd (e-mail: angela.todd@esr.cri.nz).
}

We report on the first identification of Enterovirus C105 (EV-C105) in New Zealand from a 52-year-old male hospitalized with mild respiratory tract symptoms. Enterovirus genotyping was performed by partial sequencing of the VP1 region of the enterovirus genome. This highlights the importance of enterovirus surveillance for detection of the importation of new genotypes such as EV-C105, thus allowing a better understanding of the roles they play in disease.

$\mathrm{T}$ he New Zealand National Poliovirus and Enterovirus Identification Reference Laboratory at the Institute of Environmental Science and Research (ESR) Limited, National Centre for Biosecurity and Infectious Disease routinely receives untyped enterovirus clinical specimens or cell culture isolates from four major hospitals (based in Auckland, Waikato, Wellington and Christchurch) as part of the New Zealand enterovirus surveillance network. This surveillance network started in the 1980s and on average receives 150 specimens per year. The aims of this network are (1) to identify the circulation patterns of enterovirus genotypes and predominant strains; (2) to describe clinical diseases associated with circulating genotypes; (3) to detect enterovirus outbreaks to assist with public health intervention; and finally (4) to supplement the national poliovirus surveillance programme. The four laboratories in conjunction with ESR service the entire population of New Zealand. Enterovirus surveillance operates year-round and is based on reports from routine diagnostic services for patients. Each week, all enterovirus identification results are reported to ESR and then collated and published nationally. ${ }^{1}$ In addition, untyped enteroviruses are referred to ESR for further identification.

Enterovirus C105 (EV-C105) is a member of the human enterovirus group $\mathrm{C}$ species and was first identified in the Democratic Republic of Congo in late 2010 in a faecal sample collected from a fatal acute flaccid paralysis patient during a poliomyelitis outbreak. ${ }^{2}$ Due to a lack of sequence data this virus was initially identified as Enterovirus 109 (EV-109). ${ }^{3}$ In 2012, the virus was assigned as EV-C105 because subsequent sequencing showed that it had only $74.7 \%$ nucleotide and $82.5 \%$ amino acid identity to EV-109, thus satisfying the criteria for assignment of a new enterovirus serotype. ${ }^{4}$ Since then, EV-C105 has also been identified in a patient in Peru and in another patient in Cyprus, both with respiratory disease. ${ }^{5,6}$ This suggests a global distribution although the overall incidence appears to be very low. A greater awareness of EV-C105 may help to detect this virus with increased frequency and to find out more about the pathogenic role and disease association of this particular genotype.

Here we report on the identification of EV-C105 for the first time in New Zealand from a patient with mild lower respiratory tract symptoms during an admission to a hospital in Auckland through the national enterovirus surveillance system.

\section{CASE REPORT}

A 52-year-old Maori male was admitted to a hospital in Auckland, New Zealand in October 2013. Unrelated to the reason for presenting to the hospital, cough and wheeze were noted on the second day of his admission and a nasopharyngeal swab for respiratory virus testing was collected. The patient remained

Institute of Environmental Science and Research Limited, National Centre for Biosecurity and Infectious Disease, Wallaceville, Upper Hutt, New Zealand.

Middlemore Hospital, Otahuhu, Auckland, New Zealand

Submitted: 23 October 2014; Published: 10 February 2015

doi: 10.5365/wpsar.2014.5.4.003 
afebrile. The nasopharyngeal swab tested negative for influenza, adenovirus, respiratory syncytial virus, human metapneumovirus, parainfluenza viruses $1-3$ and rhinovirus. ${ }^{7}$ Enteroviral RNA was detected using realtime reverse transcription polymerase chain reaction. ${ }^{8}$ A partial VP1 sequence was then obtained by amplification and sequencing of a $375 \mathrm{bp}$ region. ${ }^{9}$ The sequence was compared to known VP1 sequences in GenBank using tblastx (which searches a translated nucleotide database using a translated nucleotide query). ${ }^{10}$ It showed $99 \%$ amino acid identity to EV-C105 with sequence coverage of $100 \%$ (GenBank accession number: AFG25720), an EV-C105 strain that was identified in Cyprus in 2012. The next closest genotype was EV-109 with 89\% amino acid identity (GenBank accession number: ADK22861), another group $\mathrm{C}$ enterovirus.

\section{DISCUSSION}

We have reported the identification of EV-C105 for the first time in the Asia-Pacific region from a 52-year-old male with mild lower respiratory tract symptoms. EV-C105 has been identified only rarely in various countries including the Democratic Republic of Congo, Peru and Cyprus. Prior to detecting the EV-C105 case in New Zealand, EV-C105 has been previously identified in a faecal sample from an acute flaccid paralysis patient and respiratory samples taken from paediatric patients. The identification of newly-identified enteroviruses such as EV-C105 illustrates the importance of ongoing enterovirus surveillance in allowing the identification of emerging and re-emerging enterovirus genotypes and has the potential to inform public health interventions. Increased awareness of EV-C105 may allow for improved detection of the virus; however, further analysis is required to determine the pathogenic role, disease association, mode of transmission, global occurrence and public health implications of this rare enterovirus.

\section{Conflicts of interest}

None declared.

\section{Funding}

The national poliovirus/enterovirus surveillance is funded by the New Zealand Ministry of Health, which kindly permitted the use of relevant data for publication.

\section{Acknowledgements}

We wish to acknowledge W Allan Nix, research microbiologist in the Picornavirus Laboratory at the Centers for Disease Control and Prevention, for his assistance with the enteroviral typing analysis and review of this paper.

\section{References:}

1. Public Health Surveillance [Internet]. Information for New Zealand Public Health Action (https://surv.esr.cri.nz/virology/ virology_weekly_report.php, accessed 20 November 2014).

2. Grard $\mathrm{G}$ et al. Type 1 wild poliovirus and putative enterovirus 109 in an outbreak of acute flaccid paralysis in Congo, October-November 2010. Eurosurveillance: European Communicable Disease Bulletin, 2010, 15(47):pii:19723. pmid:21144443

3. Lukashev AN et al. Novel serotypes 105 and 116 are members of distinct subgroups of human enterovirus C. The Journal of General Virology, 2012, 93:2357-2362. doi:10.1099/vir.0.043216-0 pmid:22894922

4. Oberste MS et al. Molecular evolution of the human enteroviruses: correlation of serotype with VP1 sequence and application to picornavirus classification. Journal of Virology, 1999, 73:19411948. pmid: 9971773

5. Richter $\mathrm{J}$ et al. Newly emerging $\mathrm{C}$ group enteroviruses may elude diagnosis due to a divergent 5'-UTR. International Journal of Infectious Diseases, 2013, 17:e1245-1248. doi:10.1016/j. ijid.2013.07.010 pmid:24080070

6. Tokarz R et al. Genomic analysis of two novel human enterovirus $C$ genotypes found in respiratory samples from Peru. The Journal of General Virology, 2013, 94:120-127. doi:10.1099/ vir.0.046250-0 pmid:23034595

7. Centers for Disease Control and Prevention (CDC). CDC Real-Time RT-PCR Assays for Non-Influenza Respiratory Viruses. Atlanta, Centers for Disease Control and Prevention, 2010.

8. Oberste MS et al. Comparative evaluation of Taqman real-time PCR and semi-nested VP1 PCR for detection of enteroviruses in clinical specimens. Journal of Clinical Virology, 2010, 49:73-74. doi:10.1016/j.jcv.2010.06.022 pmid:20667767

9. Nix WA, Oberste MS, Pallansch MA. Sensitive, seminested PCR amplification of VP1 sequences for direct identification of all enterovirus serotypes from original clinical specimens. Journal of Clinical Microbiology, 2006, 44:2698-2704. doi:10.1128/ JCM.00542-06 pmid: 16891480

10. Altschul SF et al. Basic local alignment search tool. Journal of Molecular Biology, 1990, 215:403-410. doi:10.1016/S00222836(05)80360-2 pmid:2231712 